\title{
Effects of Comprehensive Psychological and Behavioral Intervention on Mental Symptoms, Quality of Life, and Social Function of Patients During The First Episode of Schizophrenia
}

\author{
Yuebing Zhang ( $\nabla$ yubingzhang.id@gmail.com ) \\ Jining Medical University https://orcid.org/0000-0003-2296-783X \\ Zhoumin Yin \\ Jining Medical University \\ Luning Shang \\ Jining Medical University
}

\section{Research Article}

Keywords: first episode, schizophrenia, routine nursing, comprehensive psychological and behavioral intervention, mental symptoms, QOL, SF

Posted Date: February 11th, 2021

DOl: https://doi.org/10.21203/rs.3.rs-157520/v1

License: (c) (1) This work is licensed under a Creative Commons Attribution 4.0 International License.

Read Full License 


\section{Abstract}

To analyze the effects of comprehensive psychological and behavioral intervention on mental symptoms, quality of life (QOL) and social function (SF) of patients during the first episode of schizophrenia. A total of 97 patients having the first episode of schizophrenia and who were admitted to our hospital were selected, retrospectively analyzed for clinical materials, and divided into 2 groups based on the intervention mode. The control group (CG) was routinely managed according to the psychiatric practices, whereas the observation group (OG), in accordance with the $C G$, was managed with enhanced comprehensive psychological and behavioral interventions. The scores of Positive and Negative Syndrome Scale (PANSS), Social Disability Screening Schedule (SDSS), Satisfaction with Life Scale (SWLS), Global Assessment Scale (GAS), Brief Psychiatric Rating Scale (BPRS), and disease recurrence at pre- and post-intervention were compared between the two groups. At post-intervention, scores in general psychopathological, positive, and negative symptoms, total score in PANSS, SDSS, SWLS, GAS, and BPRS were lower in the OG than that in the CG $(P<0.05)$. Further, the recurrence rate was $2.04 \%$ in the $O G$ and $25.00 \%$ in the $C G$, with statistically significant difference $(P<0.05)$. The implementation of comprehensive psychological and behavioral interventions in patients having the first episode of schizophrenia is advantageous to improve the mental symptoms, QOL, and SF and reduce the recurrence rate. Therefore, this method deserves popularization.

\section{Introduction}

Schizophrenia is a very common mental disorder with high incidence (Lichtenstein et al. 2009). To date, its specific onset mechanism is not completely explained in clinics. Patients with schizophrenia mainly have behavioral, emotional, psychological and sensory perception problems (Fromer et al. 2016, Hannon et al. 2016). If timely measures are not adopted, patients suffer impaired cognitive functions and compromised social function (SF) (Abraham and Kulhara 1987) with prolonged time.

At the current stage, patients are administered with antipsychotic drugs, which fail to improve their compromised SF even though their conditions were controlled to a certain degree (Volavka et al.1997). In the meantime, the special needs of patients with schizophrenia result in the high interruption rate during treatment, generating significant impact on the efficacy and increases healing difficulty (Goldsmith et al. 2016, Lui et al. 2016). Furthermore, majority of patients with schizophrenia have cognitive disorders, which further worsen their functional deficiency (Stanghellini et al. 2015). A previous study demonstrated that in addition to antipsychotic drugs, active nursing methods also contribute to their improved cognitive functions and return to the society as early as possible (Castro et al. 2016).

Previously, patients with schizophrenia were managed by routine psychiatric methods, which, however, are not so ideal (Bora et al. 2017), and focus more on physiological rather than psychological changes. With medical method changes in China, the biopsychosocial medical method has been extensively applied clinically (Hannon et al.2016). To improve patients' mental status and quality of life (QOL) during the first episode of schizophrenia and help them socialize early, this study is somehow innovative by 
consolidating the comprehensive psychological and behavioral interventions based on the routine nursing method.

\section{Materials And Methods \\ 2.1 Materials}

A total of 97 patients having the first episode of schizophrenia and who were admitted to our hospital were selected, retrospectively analyzed for clinical materials, and divided into two groups based on the intervention method. The control group (CG; $n=48)$, consisting of 30 males and 18 females, was routinely managed, whereas the observation group (OG; $\mathrm{n}=49$ ), comprising 32 males and 17 females, adopted a comprehensive psychological and behavioral intervention in accordance with the nursing routine. (1) Inclusion criteria are as follows: patients who complied with the Chinese Classification of Mental Disorders $₫$ diagnosis criteria of schizophrenia and were admitted to the hospital for the first time with the $\geq 6$-week length of stay and who provided (or by their family members) informed consent to participate in the study. The medical ethnic committee approved this study. (2) Exclusion criteria are as follows: patients who withdrew their participation during the process, cooperated less, or had obvious affective disorder, substance depilatory, severe organ diseases in the heart, liver, and kidneys or malignant tumors concurrently.

\subsection{Methods}

Patients in the CG were routinely managed, including enhanced safety management, life guides, health education, and sleep pattern management depending on the patients' needs.

Based on the interventions for the $C G$, patients in the $O G$ were provided with the following comprehensive psychological and behavioral interventions:

\section{a) Social skills training}

As patients with schizophrenia were stabilized after the drug treatment, nurses communicated with them to establish a nursing plan and rest system. Patients were required to dress neatly, keep themselves clean, actively and regularly carry out daily life training, such as bathing, changing of clothes, dining, making bed, washing, and rising with initiatives. They were also encouraged to participate in collective activities, by introducing themselves to others to promote self-worth and happiness. Patients were guided to greet others, go shopping and borrow things actively, participate in various entertainment activities based on their interests, such as singing, basketball, badmintons, and rope jumping, etc., or arranged to the working and entertainment room for activities such as watching TV, listening to music, playing card, dancing, practicing calligraphy, painting, and handicraft according to their interests and educational background. These activities were arranged 5 times a week, for 45-60 min each session. Based on their scores and performances, patients were rewarded spiritually or materially.

\section{b) Enhanced health education}


During the family visit at least once a week, family members were engaged in health education concerning the knowledge related to schizophrenia, so that they would understand the onset causes, course, results, family nursing methods, and social recovery methods of schizophrenia. Two health education and publicity activities were arranged every month in the forms of lectures or collective courses to correct patients' misunderstanding on the disease so that they can return to the society earlier and faster.

\section{c) Cognitive psychotherapy}

Based on cognitive theories, patients' irrational thinking was replaced by rational thinking, and interpersonal relationship was adjusted to increase their administration adherence and treatment confidence. As their acute symptoms were effectively controlled, primary psychiatry nurses provided enhanced psychological intervention by performing a collective psychological service once a week. Majority of patients with schizophrenia may have emotional problems, interpersonal relation frustrations, study and work problems, and conflicts. Therefore, nurses guided them to properly use the psychological defense mechanism and encouraged them to release their unhealthy emotions inside, speak out their ideas, face the facts positively, and obtain more robust defense capacity against mentation.

\section{d) Reinforced individual psychological service}

Nursing management and intervention were consolidated depending on the psychological problems of each patient. Through support, encouragement, leadership, interpretation, and cognitive behavior therapy, patients were guided to correctly understand the disease and rationally adjust their emotions. Their unreasonable faith inside was dug and replaced by rational faiths in order to improve unhealthy emotions and behaviors.

Both groups were managed and followed up for 6 months.

\section{Observation indexes}

\section{i. Observation indices}

(1) Positive and Negative Syndrome Scale (PANSS) was used to evaluate the severity of mental symptoms in both groups at pre-and post-intervention, which consisted of 16 items covering three aspects: psychopathological, positive, and negative symptoms. Each item is graded from 1 to 7 , and the score is positively associated with the severity of mental symptoms. The Scale's Cronbach's $a$ is 0.888 (Ghaderi et al. 2019).

(2) Social Disability Screening Schedule (SDSS) was used to evaluate SF at pre- and post-intervention. The scale consists of 10 items, graded as $0-2$ each. The results are negatively associated with SF, and the scale's Cronbach's a is 0.715 (Fowler et al. 2019). 
(3) Satisfaction with Life Scale (SWLS) was used to evaluate the QOL of the two groups at pre-and postintervention, which consisted of 12 items graded from 1 to 3 , and the score is positively associated with the QOL. The Scale's Cronbach's a is 0.789(Hinz et al. 2018).

(4) Global Assessment Scale (GAS) was used to evaluate the general conditions of both group sat preand post-intervention. Its total score is 100 , and the Cronbach's a is 0.769 (Ferreira et al. 2017). A higher score indicates better patient conditions.

(5) Brief Psychiatric Rating Scale (BPRS), consisting of 18 items graded between 1 and 7, was used to evaluate the psychiatric severity of the two groups at pre and post-intervention. Its total score ranges between 18 and 126, and the Cronbach's a is 0.922 (Cao et al. 1785). Results are negatively associated with these verity of the disease.

(6) Recurrence: Both groups were followed up for 1 year and compared and analyzed for disease recurrence.

\section{ii. Statistical method}

Statistical analysis was performed using SPSS22.0. Numerical data are expressed as mean \pm standard deviation, and comparison studies were carried out through independent sample t-test for normally distributed data and Mann-Whitney U-test non-normally distributed data. Paired test was used for preand-pro comparison in the two groups. Nominal data are expressed as (n[\%]), and comparison studies were carried out through $\mathrm{X}^{2}$ test for intergroup comparison. For all statistical comparisons, significance was defined as $\mathrm{P}<0.055$.

\section{Results}

\subsection{Comparison between the $O G$ and $C G$ according to the general materials}

The OG consisted of 32 (65.31\%) males and 17 (34.69\%) females, whereas the CG comprised 30 $(62.50 \%)$ males and $18(37.50 \%)$ females. The age range of patients in the OG was $20-50$ (mean, 30.98 \pm 2.13 ) years, disease course of 3 and 13 (mean, $5.98 \pm 0.26$ ) months, whereas those in the CG were 19$47(30.26 \pm 2.08)$ years and6 $-15(6.03 \pm 0.19)$ months, respectively. In the OG, 20 patients graduated from primary schools or below, 19 from senior high schools or colleges, and 10 from universities or above, accounting for $40.82 \%, 38.78 \%$, and $20.41 \%$ and $19(39.58 \%), 20$ (41.67\%), and 9 (18.75\%), respectively, in the CG. No statistical difference was observed in gender, average age, average disease course, and educational background between the two groups $(P>0.05$, Table 1$)$. 
Table 1

Comparison between the $\mathrm{OG}$ and the $\mathrm{CG}$ for General Materials $[\mathrm{n}(\%)] /( \pm \mathrm{s})$

\begin{tabular}{|c|c|c|c|c|c|}
\hline \multicolumn{2}{|l|}{ Materials } & \multirow{2}{*}{$\begin{array}{l}O G(n=49) \\
32(65.31)\end{array}$} & \multirow{2}{*}{$\begin{array}{l}C G(n=48) \\
30(62.50)\end{array}$} & \multirow{3}{*}{$\frac{t / X^{2}}{0.083}$} & \multirow{3}{*}{$\begin{array}{l}P \\
0.774\end{array}$} \\
\hline \multirow[t]{2}{*}{ Gender (n) } & Male & & & & \\
\hline & Female & 17(34.69) & 18(37.50) & & \\
\hline \multicolumn{2}{|l|}{ Age $(y)$} & $30.98 \pm 2.13$ & $30.26 \pm 2.08$ & 1.684 & 0.096 \\
\hline \multicolumn{2}{|c|}{ Course of disease (month) } & $5.98 \pm 0.26$ & $6.03 \pm 0.19$ & 1.079 & 0.283 \\
\hline \multicolumn{6}{|c|}{ Educational background } \\
\hline \multicolumn{2}{|c|}{ Primary school and below } & $20(40.82)$ & 19(39.58) & 0.085 & 0.968 \\
\hline \multicolumn{2}{|c|}{ Senior high school to colleges } & 19(38.78) & $20(41.67)$ & & \\
\hline \multicolumn{2}{|c|}{ Universities and above } & $10(20.41)$ & $9(18.75)$ & & \\
\hline
\end{tabular}

\subsection{Comparison between the OG and CG according to the PANSS scores}

At pre-intervention, the scores for general psychopathological, positive, and negative symptoms in the PANSS and the total score were (39.52 \pm 1.25$),(21.58 \pm 5.22),(26.02 \pm 1.28)$, and $(47.89 \pm 5.63)$ in the OG and (39.96 \pm 1.22$),(21.62 \pm 5.19),(26.09 \pm 1.26)$, and (47.92 \pm 5.62$)$ in the CG, respectively $(t=0.263$, $0.008,0.012,0.236, P \otimes 0.05$, Fig. 1).

At post-intervention, the scores for general psychopathological, positive, and negative symptoms in the PANSS and the total score in the OG were 18.02 \pm 1.05$)$, (9.21 \pm 1.05$),(8.18 \pm 0.29)$, and $(21.05 \pm 1.28)$, respectively, which were lower than those of the CG, $(24.36 \pm 1.89),(13.25 \pm 1.01),(15.32 \pm 0.96)$, and $(38.63 \pm 1.88)$, respectively $(t=9.859,10.263,11.221,6.638, P \otimes 0.05$, Fig. 2$)$.

\subsection{Comparison between the OG and CG according to the SDSS score}

Both groups attained decreased SDSS score at post-intervention (Pख0.05), which was more significant in the $0 G$, whereas the difference at pre-intervention was not significant (Pख0.05, Table 2). 
Table 2

Comparison between the $\mathrm{OG}$ and the CG for SDSSScore $( \pm s$, Score)

\begin{tabular}{|lll|}
\hline Group & Before Intervention & After Intervention \\
\hline $\mathrm{CG}(\mathrm{n}=48)$ & $27.15 \pm 2.06$ & $18.96 \pm 1.38^{\#}$ \\
\hline $\mathrm{OG}(\mathrm{n}=49)$ & $27.22 \pm 2.03$ & $13.02 \pm 0.88^{\#^{*}}$ \\
\hline$t$ & 0.169 & 25.330 \\
\hline$P$ & 0.867 & 0.000 \\
\hline $\begin{array}{l}\text { Note: \# indicates P<0.05 as compared with the conditions before intervention; *indicates } \mathrm{P}<0.05 \text { as } \\
\text { compared with the CG. }\end{array}$ & \\
\hline
\end{tabular}

\subsection{Comparison between the OG and CG according to the SWLS score}

At pre-intervention, the SWLS scores were $(31.59 \pm 2.15)$ and $(31.62 \pm 2.12)$ in the OG and CG, respectively $((t=0.069, P \otimes 0.05)$, whereas at post-intervention, they decreased to $(15.02 \pm 1.02)$ and $(22.69 \pm 1.19)$, respectively $(t=8.998, P<0.05$, Fig. 3$)$.

\subsection{Comparison between the OG and CG according to the GAS score}

At pre-intervention, the GAS scores were (17.05 \pm 3.12$)$ and $(17.09 \pm 3.09)$ in the $O G$ and $C G$, respectively $(t$ $=0.027, P>0.05)$, whereas at post-intervention, they increased to $(78.96 \pm 2.88)$ and $(52.15 \pm 1.63)$, respectively $(t=10.369, P<0.05$, Fig. 4).

\subsection{Comparison between the OG and CG according to the BPRS score}

At pre-intervention, the difference in the BPRS score was not significant between the two groups $(P>$ $0.05)$, whereas at post-intervention, the BPRS score decreased $(P<0.05)$, which was more significant in the OG $(P<0.05$, Table 3). 
Table 3

Comparison between the $\mathrm{OG}$ and the $\mathrm{CG}$ for BPRS Score $( \pm s$, Score)

\begin{tabular}{|lll|}
\hline Group & Before Intervention & After Intervention \\
\hline $\mathrm{CG}(\mathrm{n}=48)$ & $51.26 \pm 2.69$ & $30.12 \pm 2.28^{\#}$ \\
\hline $\mathrm{OG}(\mathrm{n}=49)$ & $51.32 \pm 2.58$ & $22.03 \pm 1.15^{\# \star}$ \\
\hline$t$ & 0.112 & 22.131 \\
\hline$P$ & 0.911 & 0.000
\end{tabular}

Note: \# indicates $\mathrm{P}<0.05$ as compared with the conditions before intervention; *indicates $\mathrm{P}<0.05$ as compared with the CG.

\subsection{Comparison between the OG and CG according to the recurrence rate}

After 1-year follow-up, the recurrence rate of patients in the OG was $2.04 \%$, which was lower than those in the $C G, 25.00 \%(P<0.05$, Table 4$)$.

Table 4

Comparison between the $\mathrm{OG}$ and the $\mathrm{CG}$ for Reoccurrence Rate[n(\%)]

\begin{tabular}{|lll|}
\hline Group & $\mathbf{n}$ & Reoccurrence Rate \\
\hline CG & 48 & $12(25.00)$ \\
OG & 49 & $1(2.04)^{\star}$ \\
$X^{2}$ & & 11.013 \\
\hline$P$ & & 0.000 \\
\hline Note: *indicates $P<0.05$ as compared with the CG. \\
\hline
\end{tabular}

\section{Discussion And Conclusion}

With high disability and recurrence rate, schizophrenia will lead to heavy social and family burdens and significantly affect patients' QOL (McCreadie 1997). Therefore, reducing the mental disability and slowing down SF decline play a significant role.

Due to the relatively short course and lower SF injury, patients are highly expected to recover during the first episode of schizophrenia (Jones et al. 2016). However, in real life, majority of patients and their family members greatly lack knowledge and understanding on schizophrenia, family nursing, controlling patients' conditions, and reducing the recurrence rate. Scholars including Hardingham (Hardingham and Do 2016) once conducted a study that proved that the family members' degree of knowledge on mental 
hygiene and their attitude toward the patients are the most important factors affecting the QOL of patients with schizophrenia. Therefore, patients' family members shall provide the necessary physical and psychological supports during the intervention for schizophrenia. Based on the results of this study, scores in the general psychopathological, positive, and negative symptoms in the PANSS and the total score $(P<0.05)$ were significantly lower in the $O G$ than that in the $C G$, indicating that enhanced comprehensive psychological and behavioral intervention on patients with schizophrenia can effectively improve their mental symptoms because health education and cognitive and psychological treatments are provided to consolidate patients' knowledge and understanding on the disease, correct their misunderstanding, and help them build rational faith and improve unhealthy psychological status(Fernandes et al. 2016, Grace 2016).

Though antipsychotic drugs can improve the clinical symptoms of schizophrenia to a certain degree, most of them are still related with SF deficiency (Singh et al. 2016). Thus, to improve their SF and living ability, social skills training was enhanced in this study. The results showed that SDSS and SWLS scores in the $O G$ were lower than that in the $C G$ at post-intervention $(P<0.05)$, prompting that enhanced comprehensive psychological and behavioral intervention on patients with schizophrenia resulted in significant improvement of SF and QOL consequently. The underlying reason is that the daily movement training such as bathing, changing clothes, dining, making bed, washing, and rising can significantly improve their daily living ability. Next, patients were encouraged to actively participate in entertainment activities according to their interests and habits, greet others imitatively, go shopping, and borrow things, which create conditions of being connected with the external world, to improve SF and QOL subsequently (Firth et al. 2017).

Kambeitz (Kambeitz et al. 2015) et al. revealed that no radical therapy was clinically available against schizophrenia. The core problems of patients with schizophrenia include reduced SF, cognitive function, and obvious mental symptoms. In this study, the OG had higher GAS score and lower BPRS score at postintervention $(P<0.05)$, indicating that patients' conditions were alleviated after the application of comprehensive psychological and behavioral intervention. Next, both groups were followed up for 1 year. The recurrence rates were $2.04 \%$ and $25.00 \%$ in the $O G$ and CG, respectively $(P<0.05)$, which further confirmed the effectiveness of comprehensive psychological and behavioral intervention in obviously reducing the occurrence rate. Through consolidated cognitive and psychological treatment and rational adjustment of interpersonal relation, patients' administration adherence increased and conditions were effectively controlled (Samara et al.2016). In addition, through support, encouragement, leadership, interpretation, and cognitive behavior therapy, patients were guided psychologically and individually to correctly understand the disease and rationally adjust their emotions. As a result, the recovery efficacy was dominant, and the recurrence rate was controlled at a low level.

The implementation of comprehensive psychological and behavioral intervention in patients during the first episode of schizophrenia can effectively improve patients' mental symptoms, QOL, and SF of patients and reduce the recurrence rate. Therefore, this method deserves popularization. 
However, this study included a small number of study participants; therefore, the results were not representative. In the future, a larger sample size shall be conducted for further in-depth study.

\section{Declarations}

\section{Acknowledgments}

None.

Funding: Not applicable

Conflicts of interest Statement: Not applicable

\section{Ethics approval:}

\section{Compliance with Ethical Standards}

\section{Conflict of interest}

The authors declare that they have no conflict of interest.

\section{Human and Animal Rights}

This article does not contain any studies with human or animal subjects performed by any of the authors.

\section{Informed Consent}

Informed consent was obtained from all individual participants included in the study.

Consent to participate: Not applicable

Consent for publication: Not applicable

Availability of data and material: Data sharing is not applicable to this article as no new data were created or analyzed in this study.

Code availability: Not applicable

\section{Authors' contributions}

$Y Z$ agreed on the content of the study. YZ, ZY and LS collected all the data for analysis. YZ agreed on themethodology. YZ, ZY and LS completed the analysis based on agreed steps. Results and conclusions arediscussed and written together. The author read and approved the final manuscript.

\section{References}


1. Abraham, K. R., \& Kulhara, P. (1987). The efficacy of electroconvulsive therapy in the treatment of schizophrenia: a comparative study. The British Journal of Psychiatry., 151, 152-155.

2. Bora, E., Akdede, B., \& Alptekin, K. (2017). The relationship between cognitive impairment in schizophrenia and metabolic syndrome: a systematic review and meta-analysis. Psychological medicine., 47, 1030-1040.

3. Cao, Y., Chen, X., Xie, H., Zou, L., Hu, L.-J., \& Zhou, X.-J. (1785). Correlation between electroencephalogram alterations and frontal cognitive impairment in esophageal cancer patients complicated with depression. Chinese medical journal 2017; 130.

4. Castro, E., Hjelm, R. D., Plis, S. M., Dinh, L., Turner, J. A., \& Calhoun, V. D. (2016). Deep independence network analysis of structural brain imaging: application to schizophrenia. IEEE transactions on medical imaging., 35, 1729-1740.

5. Fernandes, B., Steiner, J., Bernstein, H., Dodd, S., Pasco, J., Dean, O. M., Nardin, P., Gonçalves, C. A., \& Berk, M. (2016). C-reactive protein is increased in schizophrenia but is not altered by antipsychotics: meta-analysis and implications. Molecular psychiatry., 21, 554.

6. Ferreira, R. J., Dougados, M., Kirwan, J. R., Duarte, C., de Wit, M., Soubrier, M., Fautrel, B., Kvien, T. K., da Silva, J. A., \& Gossec, L. (2017). Drivers of patient global assessment in patients with rheumatoid arthritis who are close to remission: an analysis of 1588 patients. Rheumatology., 56, 1573-1578.

7. Firth, J., Stubbs, B., Rosenbaum, S., Vancampfort, D., Malchow, B., Schuch, F., Elliott, R., Nuechterlein, K. H., \& Yung, A. R. (2017). Aerobic exercise improves cognitive functioning in people with schizophrenia: a systematic review and meta-analysis. Schizophrenia Bulletin., 43, 546-556.

8. Fowler, D., French, P., Banerjee, R., Barton, G., Berry, C., Byrne, R., Clarke, T., Fraser, R., Gee, B., \& Greenwood, K. (2017). Prevention and treatment of long-term social disability amongst young people with emerging severe mental illness with social recovery therapy (The PRODIGY Trial): study protocol for a randomised controlled trial. Trials., 18, 315.

9. Fromer, M., Roussos, P., Sieberts, S. K., Johnson, J. S., Kavanagh, D. H., Perumal, T. M., Ruderfer, D. M., Oh, E. C., Topol, A., \& Shah, H. R. (2016). Gene expression elucidates functional impact of polygenic risk for schizophrenia. Nature Neuroscience., 19, 1442-1453.

10. Ghaderi, A., Bussu, A., Tsang, C., \& Jafarnejad, S. (2019). Retraction Note to: Effect of N-acetyl cysteine (NAC) supplementation on positive and negative syndrome scale in schizophrenia: a systematic review and meta-analysis of randomised controlled trials. European journal of clinical pharmacology., 75, 741-741.

11. Goldsmith, D., Rapaport, M., \& Miller, B. J. (2016). A meta-analysis of blood cytokine network alterations in psychiatric patients: comparisons between schizophrenia, bipolar disorder and depression. Molecular psychiatry., 21, 1696.

12. Grace, A. (2016). Dysregulation of the dopamine system in the pathophysiology of schizophrenia and depression. Nature reviews. Neuroscience., 17, 524-532.

13. Hannon, E., Spiers, H., Viana, J., Pidsley, R., Burrage, J., Murphy, T. M., Troakes, C., \& Turecki, G., O'donovan MC and Schalkwyk LC (2016). Methylation QTLs in the developing brain and their 
enrichment in schizophrenia risk loci. Nature neuroscience., 19, 48.

14. Hannon, E., Dempster, E., Viana, J., Burrage, J., Smith, A. R., Macdonald, R., St Clair, D., Mustard, C., Breen, G., \& Therman, S. (2016). An integrated genetic-epigenetic analysis of schizophrenia: evidence for co-localization of genetic associations and differential DNA methylation. Genome biology., 17, 176.

15. Hardingham, G. E., \& Do, K. Q. (2016). Linking early-life NMDAR hypofunction and oxidative stress in schizophrenia pathogenesis. Nature Reviews Neuroscience., 17, 125.

16. Hinz, A., Conrad, I., Schroeter, M. L., Glaesmer, H., Brähler, E., Zenger, M., Kocalevent, R.-D., \& Herzberg, P. Y. (2018). Psychometric properties of the Satisfaction with Life Scale (SWLS), derived from a large German community sample. Quality of Life Research., 27, 1661-1670.

17. Jones, H. J., Stergiakouli, E., Tansey, K. E., Hubbard, L., Heron, J., Cannon, M., Holmans, P., Lewis, G., Linden, D. E., \& Jones, P. B. (2016). Phenotypic manifestation of genetic risk for schizophrenia during adolescence in the general population. JAMA psychiatry., 73, 221-228.

18. Kambeitz, J., Kambeitz-llankovic, L., Leucht, S., Wood, S., Davatzikos, C., Malchow, B., Falkai, P., \& Koutsouleris, N. (2015). Detecting neuroimaging biomarkers for schizophrenia: a meta-analysis of multivariate pattern recognition studies. Neuropsychopharmacology., 40, 1742.

19. Lichtenstein, P., Yip, B. H., Björk, C., Pawitan, Y., Cannon, T. D., Sullivan, P. F., \& Hultman, C. M. (2009). Common genetic determinants of schizophrenia and bipolar disorder in Swedish families: a population-based study. The Lancet, 373, 234-239.

20. Lui, S., Liu, A., Chui, W., Li, Z., Geng, F., Wang, Y., Heerey, E., Cheung, E., \& Chan, R. (2016). The nature of anhedonia and avolition in patients with first-episode schizophrenia. Psychological medicine., 46, 437-447.

21. McCreadie, R. G. (1997). The Nithsdale Schizophrenia Surveys 16. Breast-feeding and schizophrenia: preliminary results and hypotheses. The British Journal of Psychiatry., 170, 334-337.

22. Samara, M. T., Dold, M., Gianatsi, M., Nikolakopoulou, A., Helfer, B., Salanti, G., \& Leucht, S. (2016). Efficacy, Acceptability, and Tolerability of Antipsychotics in Treatment-Resistant Schizophrenia: A Network Meta-analysis. 73: 199-210.

23. Singh, T., Kurki, M. I., Curtis, D., Purcell, S. M., Crooks, L., McRae, J., Suvisaari, J., Chheda, H., Blackwood, D., \& Breen, G. (2016). Rare loss-of-function variants in SETD1A are associated with schizophrenia and developmental disorders. Nature neuroscience., 19, 571.

24. Stanghellini, G., Ballerini, M., Presenza, S., Mancini, M., Raballo, A., Blasi, S., \& Cutting, J. (2015). Psychopathology of lived time: abnormal time experience in persons with schizophrenia. Schizophrenia bulletin., 42, 45-55.

25. Volavka, J., Laska, E., Baker, S., Meisner, M., Czobor, P., \& Krivelevich, I. (1997). History of violent behaviour and schizophrenia in different cultures: analyses based on the WHO study on determinants of outcome of severe mental disorders. The British Journal of Psychiatry., 171, 9-14.

\section{Figures}


$\rightarrow$ Observation group

$\rightarrow$ Control group

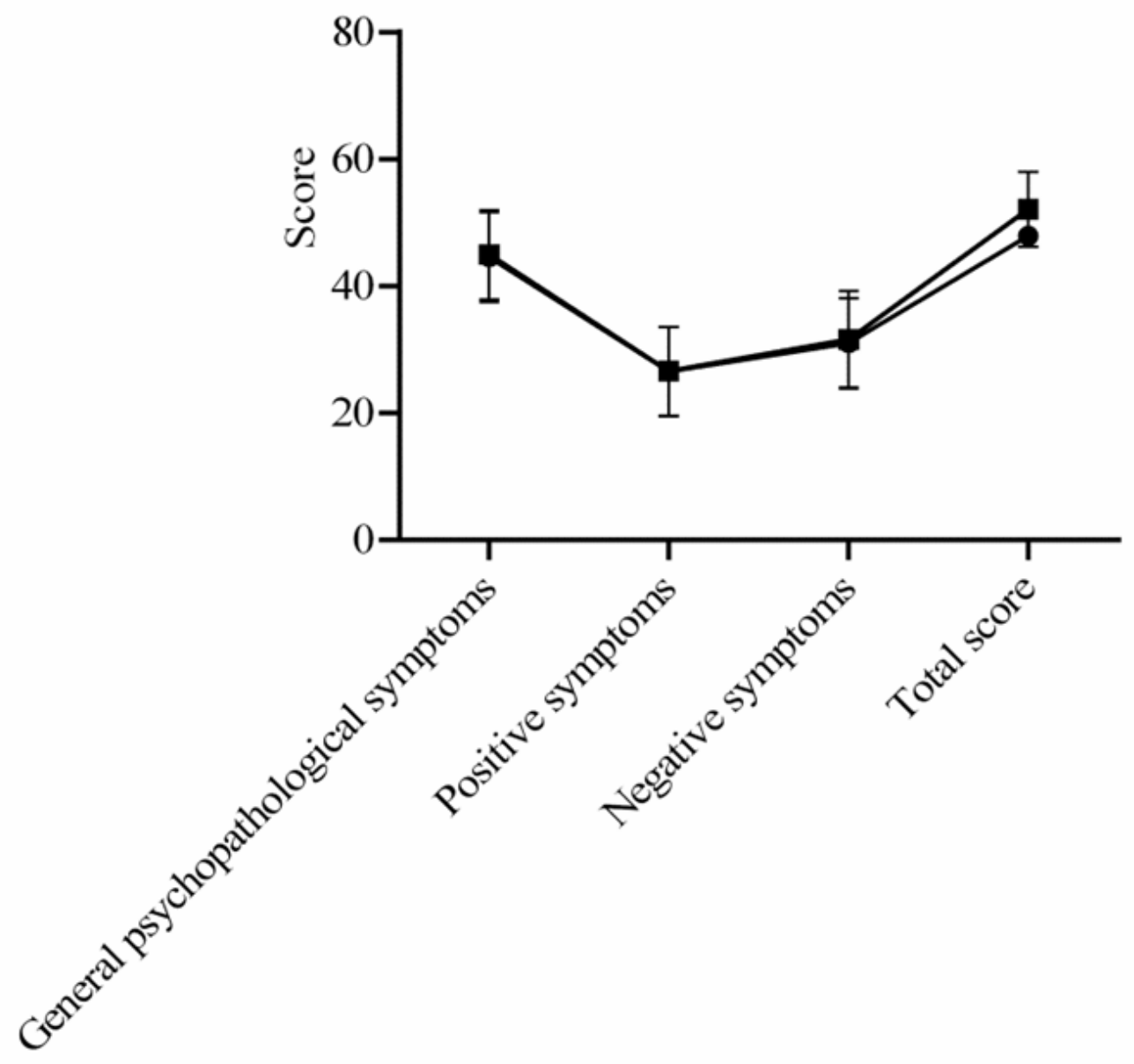

Figure 1

Comparison between the OG and the CG for PANSS Score before Intervention The 2 groups were compared for general psychopathological symptoms, positive symptoms, negative symptoms, and total score of PANSS before intervention $(P>0.05)$. 


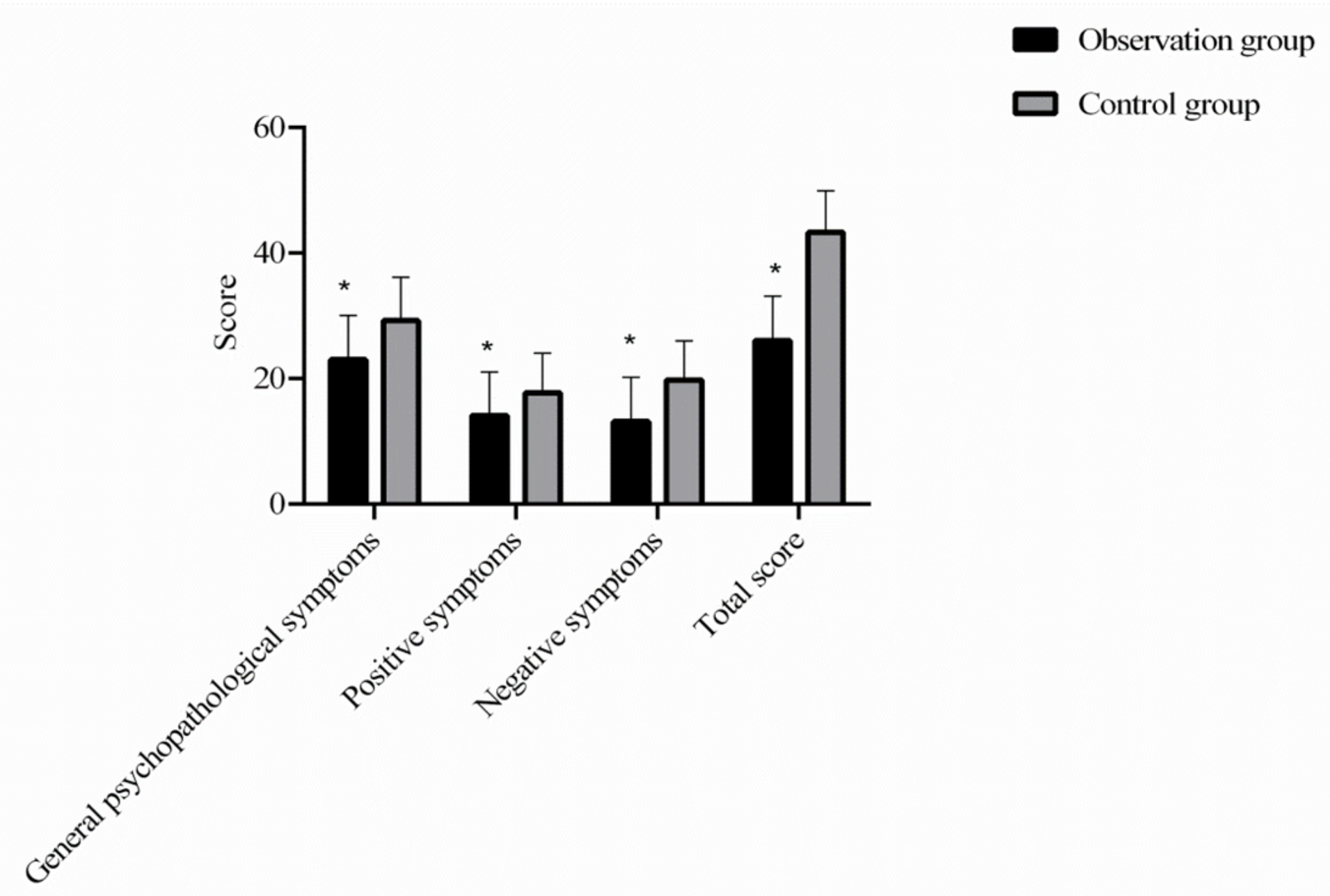

Figure 2

Comparison between the $\mathrm{OG}$ and the CG for PANSS Score After Intervention Comparison of general psychopathological symptoms, positive symptoms, negative symptoms, and total score of PANSS after intervention showed lower results in the OG than the CG $(P<0.05)$. Note: *indicates $P<0.05$ as compared with the CG. 


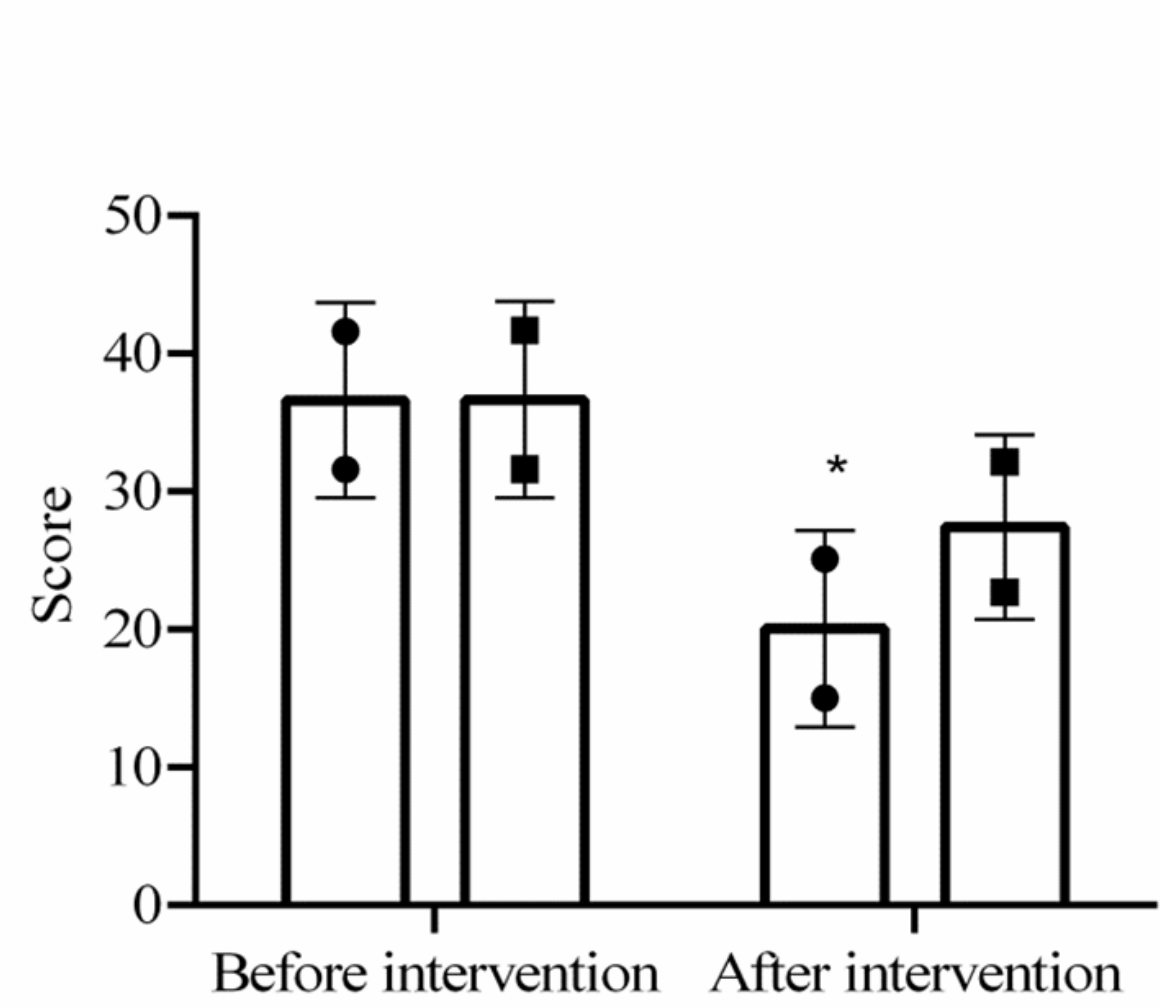

- Observation group

- Control group

Figure 3

Comparison between the OG and the CG for SWLS Score After Intervention The 2 groups were compared for SWILS scores before intervention (P凶0.05); after intervention, the OG reported lower SWLS score than the $C G(P<0.05)$. Note: *indicates $P<0.05$ as compared with the $C G$. 


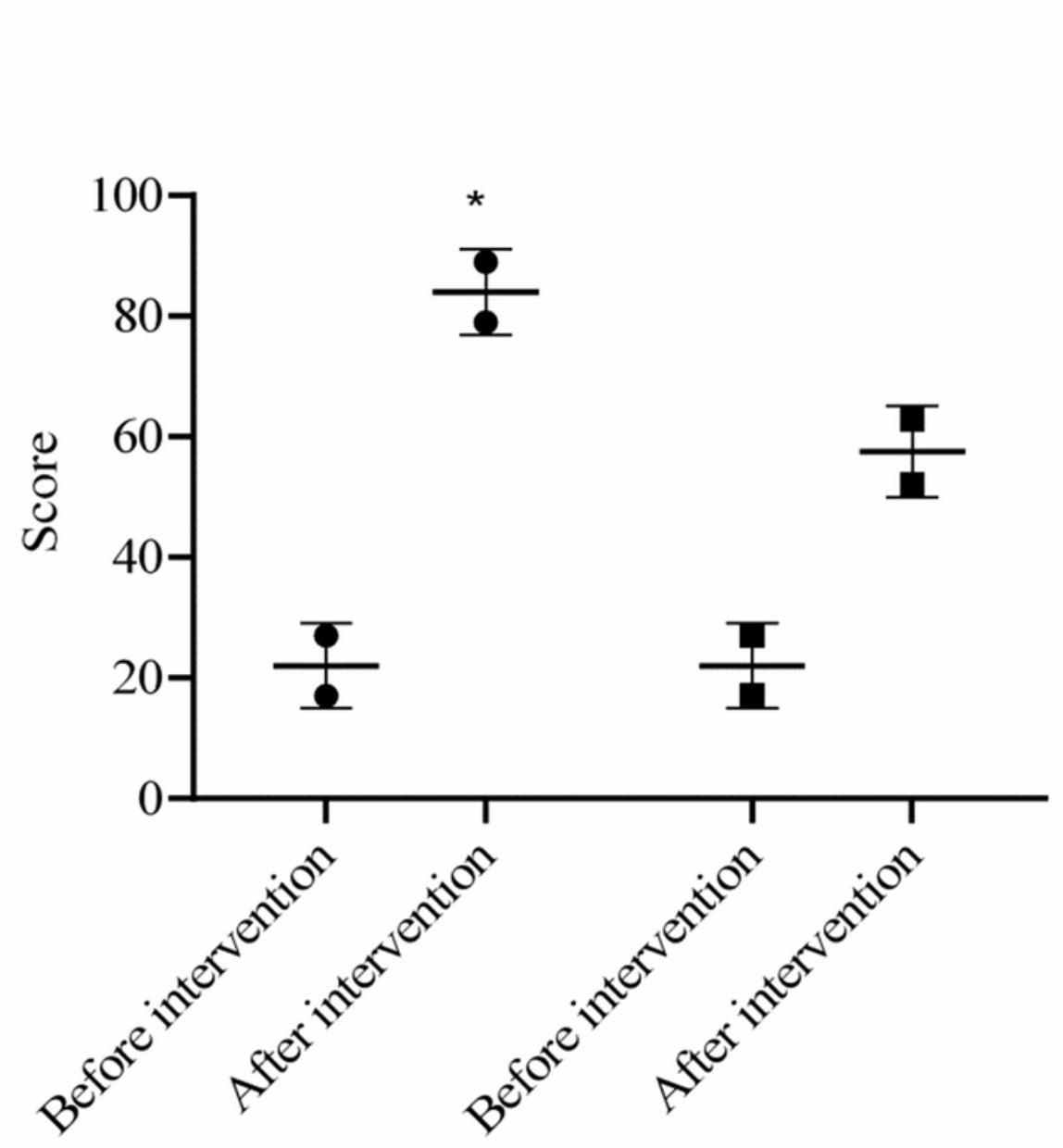

- Observation group

- Control group

Figure 4

Comparison between the $\mathrm{OG}$ and the $\mathrm{CG}$ for GAS Score After Intervention The 2 groups were compared for GAS scores before intervention (Pख0.05); after intervention, the OG reported higher GAS score than the CG $(P<0.05)$. Note: *indicates $P<0.05$ as compared with the $C G$. 